\title{
Efectos de la prótesis parcial removible sobre la salud periodontal
}

\author{
The effects of removable partial denture on periodontal health
}

ARDILA MEDINA CM*
Ardila Medina CM. Efectos de la prótesis parcial removible sobre la salud periodontal. Av Periodon Implantol. 2010; 22, 2: 77-83

\begin{abstract}
RESUMEN
Cuando no está indicada una prótesis fija, la prótesis parcial removible (PPR) puede ser el tratamiento de elección para la restauración de rebordes parcialmente edéntulos. Aun cuando la PPR es un medio para reemplazar dientes perdidos puede volverse una amenaza para los dientes remanentes de los pacientes debido a que puede incrementar la incidencia de caries, afectar el periodonto y aumentar la cantidad de fuerzas sobre los pilares. Estas alteraciones se atribuyen a una higiene oral deficiente, incremento de la placa bacteriana y acumulación de cálculos, además de la transmisión de fuerzas excesivas a las estructuras periodontales a partir de la estructura metálica de la PPR. Es posible reducir los efectos negativos de la PPR si se realizan medidas adecuadas de higiene bucal ya que solamente se han notado efectos dañinos menores en pacientes atendidos regularmente.
\end{abstract}

PALABRAS CLAVE: Prótesis parcial removible, efectos adversos.

\section{SUMIMARY}

A removable partial denture (RPD) is a common treatment available for the restoration of partially edentulous ridges. RPD although they serve as an excellent means of replacing missing teeth, may pose a serious threat to a patient's remaining teeth. RPD may increase incidence the incidence of caries, damage the periodontium, and increase the amount of stress on natural teeth. These alterations are attributed to poor oral hygiene, increased plaque and calculus accumulation, and transmission of excessive forces to the periodontal structures from occlusal surfaces of the framework of RPD. It is possible to reduce the negative effects of removable partial dentures on the periodontium and perform good oral hygiene. Only minor periodontal effects were noted in patients recalled regularly for supportive treatment, including professional oral hygiene.

KEY WORDS: Denture partial removable, adverse effects.

Fecha de recepción: 14 de enero de 2009.

Fecha de aceptación: 30 de enero de 2009.

\section{INTRODUCCIÓN}

Los hallazgos de la literatura relacionados con la prótesis parcial removible (PPR) y sus implicaciones periodontales presentan resultados contradictorios. Algunas investigaciones que han evaluado el efecto de la PPR sobre la salud gingival y periodontal demuestran que los dientes contenidos en su diseño desa- rrollan significativamente más enfermedad periodontal comparándolos con aquellos que no están incluidos (1-5). Algunos autores han sugerido que el uso de PPR conduce a cambios importantes en la cantidad y calidad de la formación de la placa bacteriana alrededor de los dientes remanentes $(6,7)$. Por otra parte, varios investigadores han concluido que cuando se realiza una buena higiene bucal se presenta 
poco o ningún daño sobre los dientes remanentes y su soporte periodontal $(2,8)$. El objetivo de este artículo es presentar los posibles efectos que puede ocasionar la presencia de una PPR sobre la salud periodontal.

\section{EFECTO SOBRE LA PLACA BACTERIANA, LA PROFUNDIDAD DE SONDAJEY EL NIVEL DE INSERCION}

Diferentes estudios han mostrado el efecto de la PPR sobre la calidad y la cantidad de la placa bacteriana. Se evaluó la formación de placa sobre 44 dientes pilares de PPR mientras los sujetos usaban o no sus prótesis (9). Se encontró que el uso de la PPR incrementó la formación de placa sobre todas las superficies de los pilares objeto de investigación. El mismo investigador, realizó un estudio con el fin de evaluar la composición de la placa bacteriana formada sobre 15 dientes pilares en nueve pacientes parcialmente edéntulos (10). Se examinaron las bacterias localizadas en las muestras de placa formada sobre las superficies vestibulares y proximales contiguas a los espacios edéntulos. Se encontró que la presencia de PPR favorecía la proliferación de espirilos y espiroquetas, alterando la composición de la placa bacteriana. Yeung y colaboradores (11), examinaron la salud periodontal en 87 sujetos que recibieron PPR cinco años antes de la iniciación del estudio. En todos los pacientes se detectó placa bacteriana, sangrado al sondaje y pérdida de inserción. Aquellos sitios del diente que hacían contacto con la PPR presentaban más placa $(\mathrm{p}<0,001)$ y la mayoría de ellos $(63 \%)$ tenían al menos un sitio con una profundidad de sondaje mayor a $4 \mathrm{~mm}$. Se llevó a cabo un seguimiento en 36 pacientes durante 30 meses con el fin de evaluar el efecto de la plancha lingual, como conector de PPR sobre la salud periodontal, cuando se comparaba con la barra lingual (4). Los investigadores encontraron un índice de placa (12) mayor en el grupo con plancha lingual $(\mathrm{p}<0,05)$, así como también mayor recesión gingival. Un total de 205 pacientes que usaban PPR participaron en un estudio con el fin de evaluar su efecto sobre la salud periodontal de dientes pilares y no pilares (2). Los sujetos habían tenido sus prótesis por periodos entre 1 y 10 años. La mayor parte de pilares naturales $(70 \%)$ presentaron índices de placa (12) elevados. El 54\% de los pilares naturales presentaron el valor promedio más alto para la profundidad de sondaje ( 3 a $5 \mathrm{~mm}$ ) y el promedio más elevado de recesión gingival ( $2 \mathrm{~mm}$ ). Los pilares con coronas presentaron resultados similares a los arrojados por los pilares naturales $(p>0,05)$.
Addy y colaboradores (13), estudiaron 46 PPR y su efecto sobre la acumulación de placa bacteriana, concluyendo que en los sujetos que usan PPR es fundamental emplear medidas estrictas de higiene oral, recomendando además diseños protésicos simples. Varios estudios clínicos han concluido que un adecuado control de placa en pacientes portadores de PPR requiere la implementación de programas estrictos de mantenimiento y excelentes medidas en higiene oral (14-17).

La tabla l presenta algunos estudios clínicos y los efectos de la PPR sobre la salud periodontal.

\section{EFECTO SOBRE LA MOVILIDAD DENTAL}

Diferentes publicaciones han sugerido que el diseño de la PPR afecta la distribución de las fuerzas sobre los dientes pilares y los rebordes alveolares residuales (18-20). Sin embargo, la mayoría de estudios incluyen modelos de laboratorio y no hay un consenso claro en el momento de considerar un diseño ideal de PPR. Los autores están de acuerdo en que la rigidez de los conectores mayores y el máximo cubrimiento de las áreas de soporte con las bases protésicas son de gran importancia para reducir las fuerzas sobre los dientes pilares (21-23). Un grupo de investigadores (24), midieron las fuerzas horizontales laterales aplicadas a los dientes pilares durante el funcionamiento de PPR, demostrando que las fuerzas ejercidas sobre ellos durante la deglución son casi el doble de las desplegadas durante la masticación. Ogata y colaboradores (25), analizaron longitudinalmente el torque transmitido desde la base de la dentadura hacia los pilares. Sus hallazgos indican que no solamente las fuerzas oclusales sino también la lengua, los carrillos y los labios contribuyen a generar torque y fuerzas adicionales sobre los dientes pilares. Se midió intraoralmente la movilidad dentaria de los dientes pilares empleando dos diseños diferentes de PPR a extensión distal mandibular y con anclaje en los caninos (26). El primer diseño presentaba un apoyo meso-oclusal mientras que el segundo no lo mostraba. Los dos tipos de PPR estudiados produjeron cambios agudos, graduales y significativos sobre la movilidad dental de los pilares. Un estudio longitudinal evaluó durante 4 años sujetos que usaban PPR (27). Los autores encontraron movilidad en el $18 \%$ de los dientes presentes, mientras que en aquellos pacientes que por alguna razón no emplearon sus PPR no se encontró movilidad. Se realizó una investigación con el fin de evaluar el gra- 


\section{TABLA 1.- ESTUDIOS CLÍNICOS Y EFECTOS DE LA PPR SOBRE LA SALUD PERIODONTAL}

\begin{tabular}{|c|c|c|c|c|}
\hline Estudio & Tipo & Período & Sujetos & Efecto de la PPR \\
\hline Yusof y col. (1) & Retrospectivo & 5 años & 427 & $\begin{array}{l}\text { Aumento placa bacteriana } \\
\text { Inflamación gingival } \\
\text { Movilidad dental }\end{array}$ \\
\hline Zlatariæ y col. (2) & Retrospectivo & 10 años & 205 & $\begin{array}{l}\text { Aumento placa bacteriana } \\
\text { Incremento profundidad de sondaje } \\
\text { Recesión gingival }\end{array}$ \\
\hline Budtz-Jörgensen (3) & Prospectivo & 5 años & 31 & $\begin{array}{l}\text { Gingivitis } \\
\text { Periodontitis }\end{array}$ \\
\hline Akaltan y col. (4) & Prospectivo & 30 meses & 36 & $\begin{array}{l}\text { Acumulación de placa } \\
\text { Incremento movilidad dental } \\
\text { Recesión gingival }\end{array}$ \\
\hline Kern y col. (5) & Retrospectivo & 10 años & 74 & $\begin{array}{l}\text { Aumento profundidad de sondaje } \\
\text { Incremento movilidad dental }\end{array}$ \\
\hline Vanzeveren y col. (7) & Prospectivo & 2 años & 30 & Elevados niveles de placa bacteriana \\
\hline Yeung y col. $(11,44)$ & Retrospectivo & 5 años & 87 & $\begin{array}{l}\text { Aumento placa bacteriana } \\
\text { Sangrado al sondaje } \\
\text { Pérdida de inserción }\end{array}$ \\
\hline Bergman y col. (14) & Prospectivo & 25 años & 30 & Ninguno \\
\hline Bassi y col (15) & Corte transversal & - & 57 & Incremento placa bacteriana \\
\hline Kafur y col. (16) & Prospectivo & 5 años & 122 & Ninguno \\
\hline Mojon y col. (17) & Corte transversal & - & 120 & $\begin{array}{l}\text { Gingivitis } \\
\text { Periodontitis }\end{array}$ \\
\hline Piwowarczyk y col. (21) & Retrospectivo & 5 años & 97 & Ninguno \\
\hline Carlsson y col. (27) & Prospectivo & 4 años & 88 & Incremento de la movilidad dental \\
\hline
\end{tabular}

do y la cantidad de movimiento de los pilares distales y la base protésica producidos por el retenedor directo de extensión distal de una PPR y la localización de la carga funcional (28). Los resultados revelaron que el tipo de retenedor directo influye más sobre la magnitud que la dirección de desplazamiento de los dientes pilares. Por otra parte, la localización de los puntos de carga influye tanto en la magnitud como en la dirección del movimiento de los dientes pilares y la base protésica. Las cargas posteriores y linguales ocasionan un desplazamiento distal significativo de los pilares $(p<0,05)$. Jin y colaboradores $(29)$, exploraron los efectos de diferentes posiciones de carga sobre los movimientos de los dientes pilares y la base pro- tésica de PPR con diseños unilaterales y bilaterales. Los movimientos de los dientes pilares y la base protésica de PPR con diseño unilateral fueron significativamente mayores durante las posiciones de carga bucal, lingual y centro distal producidos en PPR. Kawata y colaboradores (30), midieron las fuerzas tridimensionales ejercidas sobre los dientes pilares de PPR durante la masticación empleando un mecanismo piezoeléctrico. El mecanismo fue localizado en el segundo bicúspide inferior derecho de un sujeto edéntulo superior. Cuando no se usaba la PPR la magnitud de la fuerza fue mayor y su dirección más posterior. La dirección fue más posterior con una PPR con un apoyo distal solamente y mas anterior con un apoyo mesial 
único. Los autores concluyeron de esta forma que las fuerzas tridimensionales ejercidas sobre los dientes pilares de una PPR depende de la localización de los apoyos.

Diferentes estudios clínicos longitudinales han demostrado que PPR con diseños adecuados no tienen ningún efecto sobre la movilidad dental siempre y cuando se realicen controles estrictos de higiene oral dentro de un programa de mantenimiento frecuente (14-16). Adicionalmente, y con el fin de garantizar una mejor distribución de las fuerzas, algunos autores sugieren la ferulización de los pilares primarios en PPR de extensión distal $(27,31,32)$.

\section{FERULIZACIÓN DE LOS DIENTES PILARES}

Carlsson y colaboradores (27), sugirieron ferulizar los pilares primarios para soportar las fuerzas ejercidas por las PPR. Su recomendación se basó en el seguimiento realizado durante 4 años en donde no se observó ningún tipo de deterioro periodontal de los pilares ferulados. Un estudio realizado sobre un modelo fotoeslástico informó que la ferulización es un aspecto importante cuando se emplean retenedores en PPR de extensión distal (32). Se concluyó además, que en pilares únicos, los retenedores inducen fuerzas distales sobre los dientes produciendo fuerzas horizontales desfavorables. Charkawi y Wakad (33), estudiaron las fuerzas inducidas alrededor de los pilares y el área de extensión distal bajo diferentes cargas como una función del número de pilares ferulados. La reducción en la cantidad de unidades feruladas de tres a dos ocasionó un incremento insignificante en las fuerzas, mientras que la disminución de dos a uno produjo un incremento significativo de ellas (nueve veces mayor). Basado en este modelo in vitro, al menos dos dientes de cada lado deben ferularse cuando se emplean retenedores extracoronales de extensión distal. Otros investigadores han encontrado resultados similares empleando análisis de elementos finitos y modelos fotoelásticos $(34,35)$.

Hasta la fecha no se conocen investigaciones que comparen pirales ferulados y no ferulados cuando se emplean PPR. Las publicaciones han concluido que en ausencia de placa bacteriana e inflamación, las fuerzas no producen pérdida de inserción sobre los dientes, no obstante, se puede incrementar lo movilidad dental como resultado de cambios adaptativos, no pa- tológicos (36-39). En el momento de realizar una PPR es fundamental tener en cuenta los principios fundamentales en su diseño incluyendo el ajuste adecuado de la estructura metálica y la construcción de bases de extensión distal empleando modelos alterados, con el fin de disminuir las fuerzas ejercidas sobre los pilares (40-43).

La figura 1 presenta un diseño inadecuado de PPR que favorece la acumulación de placa bacteriana y la movilidad dental.

\section{MANTENIMIENTO PROTÉSICO PERIODONTAL}

Diferentes estudios han hecho énfasis en la importancia del mantenimiento protésico periodontal de los pacientes que usan PPR (11, 15, 17, 44, 45). Mojon y colaboradores (17), evaluaron la influencia de la PPR sobre la enfermedad periodontal en una muestra de pacientes hospitalizados. Además del desinterés por realizar una higiene bucal adecuada, encontraron que la mayoría de pacientes habían perdido el contacto con sus odontólogos y tenían PPR defectuosas que comprometían el estado periodontal. Otro estudio (15), examinó un grupo de pacientes que habían usado PPR durante un periodo comprendido entre 6 y 12 años. Esta investigación mostró que la mayoría de pacientes fueron incapaces de mantener estándares altos de higiene oral y la condición periodontal de los dientes pilares fue peor que la de los no pilares. Los autores concluyeron que en ausencia de un programa de mantenimiento regular, solamente el $10,5 \%$ de los pacientes habían conseguido una higiene óptima. En una investigación que arrojó dos publicaciones $(11,45)$, se evaluaron 87 pacientes que habían usado PPR durante

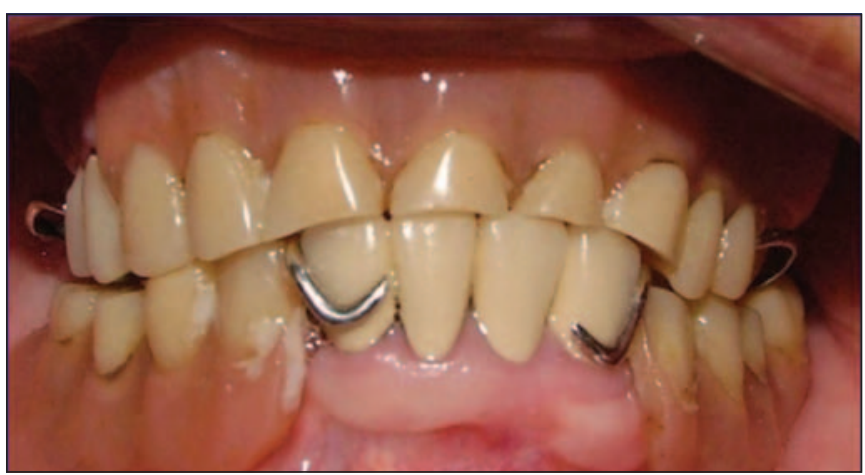

Fig. 1: Diseño inadecuado de PPR que favorece la acumulación de placa bacteriana y la movilidad dental. 
$5 \circ 6$ años. Los investigadores observaron una prevalencia alta de placa bacteriana, gingivitis asociada a placa y recesiones gingivales, recomendando por lo tanto, refuerzos regulares en higiene oral, detartraje y profilaxis.

La frecuencia del período de mantenimiento deben diseñarse de acuerdo a las características y necesidades individuales del paciente y también según su habilidad para realizar controles adecuados de placa bacteriana, teniendo en cuenta también el mantenimiento protésico $(11,17,45)$.

\section{CONCLUSIONES}

La utilización de una PPR conduce a cambios en la cantidad y calidad de la placa bacteriana, por ello, la ejecución de medidas adecuadas de higiene es fundamental para reducirlos.

Los factores que afectan la distribución de las fuerzas de la PPR a los dientes pilares y reborde edéntulo abarcan el diseño protésico, la adaptación de la base protésica y la inclinación del reborde residual.

Está indicada la ferulización de los dientes pilares cuando está reducido el soporte periodontal o se espera un aumento de las fuerzas, como cuando se emplean retenedores intracoronales.

Es fundamental un diseño protésico adecuado que evite efectos dañinos sobre las estructuras periodontales remanentes.

El mantenimiento protésico periodontal es indispensable para lograr un buen pronóstico a largo plazo.

\section{BIBLIOGRAFÍA}

1. Yusof Z, Isa Z. Periodontal status of teeth in contact with denture in removable partial denture wearers. J Oral Rehabil 1994; 21:77-86.

2. Zlatariæ DK, Celebiæ A, Valentiæ-Peruzoviæ M. The effect of removable partial dentures on periodontal health of abutment and non-abutment teeth. J Periodontol 2002;73:137-44.

3. Budtz-Jörgensen E. Effects of denture-wearing habits on periodontal health of abutment teeth in patients with overdentures. J Clin Periodontol 1994;21:265-9
4. Akaltan F, Kaynak D. An evaluation of the effects of two distal extension removable partial denture designs on tooth stabilization and periodontal health.J Oral Rehabil 2005;32:823-9.

5. Kern M, Wagner B. Periodontal findings in patients 10 years after insertion of removable partial dentures. J Oral Rehabil 2001;28:991-7.

6. Petridis H, Hempton TJ. Periodontal considerations in removable partial denture treatment: $A$ review of the literature. Int J Prosthodont 2001;14:164-72.

7. Vanzeveren C, D'Hoore W, Bercy P. Influence of removable partial denture on periodontal indices and microbiological status. J Oral Rehabil 2002;29:232-9.

8. Zlatariæ DK, Celebiæ A, Valentiæ-Peruzoviæ M, Jerolimov V, Panduriæ J. A survey of treatment outcomes with removable partial dentures. J Oral Rehabil 2003; 30: 847-54.

9. Ghamrawy EE. Quantitative changes in dental plaque formation related to removable partial dentures. J Oral Rehabil 1976;3:115-20.

10. Ghamrawy EE. Qualitative changes in dental plaque formation related to removable partial dentures. J Oral Rehabil 1979; 6: 183-8.

11. Yeung AL, Lo EC, Chow TW, Clark RK. Oral health status of patients 5-6 years after placement of cobaltchromium removable partial dentures. J Oral Rehabil 2000;27:183-9.

12. Silness $P$, Loe H. Periodontal disease in pregnancy. Acta Odontol Scand 1964; 22:121.

13 Addy M, Bates JF. Plaque accumulation following the wearing of different types of removable partial dentures. J Oral Rehabil 1979;6:111-17.

14. Bergman B, Hugoson A, Olsson C-O. A 25-year longitudinal study of patients treated with removable partial dentures. J Oral Rehabil 1995;22:595-99.

15. Bassi F, Mantecchini S, Carossa S, Preti G. Oral conditions and aptitude to receive implants in patients with removable partial dentures: A cross-sectional study. Part I. Oral conditions. J Oral Rehabil 1996;23:50-4.

16. Kapur KK, Deupree R, Dent RJ, Hasse AL. A randomized clinical trial of two basic removable partial denture 
designs. Part I: Comparisons of five-year success rates and periodontal health. J Prosthet Dent 1994;72:26882.

17. Mojon P, Rentsch A, Budtz-Jörgensen E. Relationship between prosthodontic status, caries, and periodontal disease in a geriatric population. Int J Prosthodont 1995; 8:564-71.

18. Ko SH, McDowell GC, Kotowicz WE. Photoelastic stress analysis of mandibular removable partial dentures with mesial and distal rests. J Prosthet Dent 1986;56:454-60.

19. Feingold GM, Grant AA, Johnson W. The effect of partial denture design on abutment tooth and saddle movement. J Oral Rehabil 1986;13:549-57.

20. Igarashi Y, Ogata A, Kuroiwa A, Wang CH. Stress distribution and abutment tooth mobility of distalextension removable partial dentures with different retainers: An in vivo study. J Oral Rehabil 1999;26:11116.

21. Piwowarczyk A, Köhler KC, Bender R, Büchler A, Lauer $\mathrm{HC}$, Ottl P. Prognosis for abutment teeth of removable dentures: a retrospective study. J Prosthodont. 2007;16: 377-82.

22. Wöstmann B, Balkenhol M, Weber A, Ferger P, Rehmann P. Long-term analysis of telescopic crown retained removable partial dentures: survival and need for maintenance. J Dent 2007;35:939-45.

23. Nickenig HJ, Spiekermann H, Wichmann M, Andreas SK, Eitner S. Survival and complication rates of combined tooth-implant-supported fixed and removable partial dentures. Int J Prosthodont 2008;21:131-7.

24. Kydd WL, Dutton DA, Smith DW. Lateral forces exerted on abutment teeth by partial dentures. J Am Dent Assoc 1964;68:859-63.

25. Ogata K, Ishii A, Nagare I. Longitudinal study on torque transmitted from a denture base to abutment tooth of a distal extension removable partial denture with circumferential clasps. J Oral Rehabil 1992;19:245-52.

26. Fenner W, Gerber A, Mühlemann HR. Tooth mobility changes during treatment with partial denture prosthesis. J Prosthet Dent 1956;6:520-25.

27. Carlsson GE, Hedegard B, Koivumaa KK. Studies in partial denture prosthesis IV. Final results of a 4-year longitudinal investigation of dentogingivally supported partial dentures. Acta Odontol Scand 1965;23:443-69.

28. Mizuuchi W, Yatabe M, Sato M, Nishiyama A, Ohyama T. The effects of loading locations and direct retainers on the movements of the abutment tooth and denture base of removable partial dentures. J Med Dent Sci 2002;49: 11-8.

29. Jin X, Sato M, Nishiyama A, Ohyama T. Influence of loading positions of mandibular unilateral distal extension removable partial dentures on movements of abutment tooth and denture base. J Med Dent Sci 2004;51:155-63.

30. Kawata T, Kawaguchi T, Yoda N, Ogawa T, Kuriyagawa T, Sasaki K. Effects of a removable partial denture and its rest location on the forces exerted on an abutment tooth in vivo. Int J Prosthodont 2008;2 1:50-2.

31. Goodkind RJ. The effects of removable partial dentures on tooth mobility: A clinical study. J Prosthet Dent 1973;30:139-46.

32. Kratochvil FJ, Thompson WD, Caputo AA. Photoelastic analysis of stress patterns on teeth and bone with attachment retainers for removable partial dentures. J Prosthet Dent 1981;46:21-8.

33. el Charkawi HG, el Wakad MT. Effect of splinting on load distribution of extracoronal attachment with distal extension prosthesis in vitro. J Prosthet Dent 1996;76: 315-20.

34. Zheng YL, Chen L, Zhang FQ, Wang CT. Effect of the number of splinted abutments on stress distribution of support tissue. Shanghai Kou Qiang Yi Xue 1999;8:101-3.

35. Itoh H, Caputo AA, Wylie R, Berg T. Effects of periodontal support and fixed splinting on load transfer by removable partial dentures.J Prosthet Dent 1998;79:46571 .

36. Wagenberg BD. Considerations in treatment planning of the periodontal patient. Dent Today 2005;24:108-14.

37. Bernal G, Carvajal JC, Muñoz-Viveros CA. A review of the clinical management of mobile teeth. J Contemp Dent Pract 2002;3:10-22.

38. Davies SJ, Gray RJ, Linden GJ, James JA. Occlusal considerations in periodontics. Br Dent J 2001;191:597604. 
39. Svanberg GK, King GJ, Gibbs CH. Occlusal considerations in periodontology. Periodontol 2000 1995;9:106-17.

40. McCord JF, Grey NJ,Winstanley RB, Johnson A. A clinical overview of removable prostheses: 3 . Principles of design for removable partial dentures. Dent Update 2002;29:474-81.

41. Fejérdy P, Borbély J, Schmidt J, Jánh M, Hermann P. Removable partial denture design and its effect on remaining teeth, based on Hungarian national survey. Fogorv Sz 2008;101:3-11.

42. Beaumont AJJr. An overview of esthetics with removable partial dentures. Quintessence Int 2002;33:747-55.

43. McCord JF, Grey NJ, Winstanley RB, Johnson A. A clinical overview of removable prostheses: 1 . Factors to consider in planning a removable partial denture. Dent Update 2002;29:376-81.

44. Yeung AL, Lo EC, Clark RK, Chow TW. Usage and status of cobalt-chromium removable partial dentures 5-6 years after placement. J Oral Rehabil 2002;29:127-32.

45. Gorai S, Koyama S, Chiba T, Ogawa T, Hatori K, Sasaki K. Multivariate analysis of factors affecting the status of wearing removable partial dentures. Nihon Hotetsu Shika Gakkai Zasshi 2008;52:126-34.

\section{CORRESPONDENCIA}

Carlos Martín Ardila Medina

Carrera 47 No. 20 sur 46 Envigado Antioquia Colombia 57(4) 3348122

cmartin@odontologia.udea.edu.co 